\title{
Aggregate context effects in music processing
}

\section{Mark A. Schmuckler ${ }^{1}$ (D) Dominique T. Vuvan ${ }^{2} \cdot$ Olivia Podolak Lewandowska $^{1}$}

Published online: 12 March 2020

(C) The Psychonomic Society, Inc. 2020

\begin{abstract}
Control of stimulus confounds is an ever-present, and ever-important, aspect of experimental design. Typically, researchers concern themselves with such control on a local level, ensuring that individual stimuli contain only the properties they intend for them to represent. Significantly less attention, however, is paid to stimulus properties in the aggregate, aspects that, although not present in individual stimuli, can nevertheless become emergent properties of the stimulus set when viewed in total. This paper describes two examples of such effects. The first (Case Study 1) focuses on emergent properties of pairs of to-be-performed tones on a piano keyboard, and the second (Case Study 2) focuses on emergent properties of short, atonal melodies in a perception/memory task. In both cases these sets of stimuli induced identifiable tonal influences despite being explicitly created to be devoid of musical tonality. These results highlight the importance of monitoring aggregate stimulus properties in one's research, and are discussed with reference to their implications for interpreting psychological findings quite generally.
\end{abstract}

Keywords Music perception · Tonality $\cdot$ Piano performance $\cdot$ Musical memory

\section{Introduction}

One of the axiomatic rules of teaching students to conduct research is training on how to control our experimental contexts to eliminate unwanted influences from confounding factors. From a methodological viewpoint, much of the "skill" of a researcher lies in his or her ability to create controlled experimental environments that nevertheless encapsulate critical features of the real-world phenomena under investigation. However, instantiating such control requires researchers to make assumptions regarding the active influences within an experimental context, with such assumptions varying in how consciously they are adopted by researchers.

This manuscript describes one such experimental assumption, exploring the impact of abstract global stimulus parameters on participants' responses in two different experimental designs. Both of these "case studies" were

Mark A. Schmuckler

mark.schmuckler@utoronto.ca

1 Department of Psychology, University of Toronto Scarborough, 1265 Military Trail, Toronto, ON M1C 1A4, Canada

2 Department of Psychology Skidmore College, Saratoga Springs, NY, USA conducted within the field of music cognition and performance, and both demonstrated an unconscious influence of the common structural factor of musical tonality. One of the studies focused on manual behavior during simple piano performance, whereas the other study explored expectancy formation in melodic memory.

To appreciate the importance of the factor of tonality, a short discussion is in order (see Krumhansl, 1990, or Schmuckler, 2004, 2016, for reviews). In Western tonal music, tonality refers to the organization of the 12 tones of the chromatic scale (the complete set of tones) around a single reference pitch (called the tonic). Tonality induces a hierarchical structure such that some tones are perceived as closely related to the tonic and tonality, whereas other tones are perceived as unrelated to the tonality. Tonality is further structured by two principles. First, tonalities can be built using any of the 12 notes of the chromatic set as the tonic, with the corresponding hierarchical structure shifted to reflect this new tonic (Krumhansl \& Kessler, 1982). Second, in Western music there exist two forms of hierarchical structure called "major" and "minor" tonalities, differing in terms of which tones lie at the varying hierarchical levels (other musical cultures contain different types of hierarchical structuring). Combining these two principles produces 24 different tonal structures (12 major plus 12 minor tonalities, although see Vuvan, Prince, \& Schmuckler, 2011) employed in Western tonal music. 
On a psychological level, there exists a significant amount of work dedicated to modelling the psychological organization of tonality (e.g., Chew, 2014; Krumhansl \& Kessler, 1982; Tillman, Bharucha, \& Bigand, 2000). This work has demonstrated that tonality influences multiple aspects of musical behavior, including the perceptual organization of music (Krumhansl \& Schmuckler, 1986b; Thompson \& Mor, 1992), on-line processing of music (Bharucha \& Stoeckig, 1986; Bigand \& Pineau, 1997; Schmuckler \& Boltz, 1994), memory for music (Albouy, Schulze, Caclin, \& Tillman, 2013; Halpern, Bartlett, \& Dowling, 1998; Vuvan, Podolak, \& Schmuckler, 2014), and music performance in practiced (Palmer \& van de Sande, 1993), novel (Jebb \& Pfordresher, 2016; Lewandowska \& Schmuckler, 2019), and improvised (Schmuckler, 1989, 1990) contexts. Accordingly, finding that tonality plays an unintentional role in music tasks that were intended to be devoid of tonal influences would not be surprising.

The current report describes two examples of such general context effects in two experimental projects employing widely divergent music processing tasks. Because both of these projects have been previously published (Schmuckler \& Bosman, 1997; Vuvan et al., 2014), the core experiments themselves will not be presented in detail, but rather will be presented as a pair of case studies, with readers invited to consult the original publications for methodological details. The first of these case studies (Schmuckler \& Bosman, 1997) presents a series of subsidiary analyses of the original published data, whereas the second case study (Vuvan et al., 2014) presents the findings produced by an initial, pilot project on this topic using the same paradigm.

Before describing the case studies, however, it is worth considering whether the issue of generalized context effects such as these is a concern specific to research in music cognition and performance, or is applicable generally in psychological research. As a short response to this question, there is by now a well-established literature demonstrating that global context effects can and do exist across multiple areas of psychological investigation. In fact, there are many examples of the abstraction of global properties out of stimulus sets, including Posner's classic work on prototype formation (e.g., Posner, Goldsmith, \& Welton, 1967; Posner \& Keele, 1968), sensitivity to hierarchical versus non-hierarchical serial patterns (e.g., Lewkowicz, Schmuckler, \& Mangalindan, 2018; Restle, 1970), along with an array of paradigms demonstrating general context effects, such as work on hysteresis (e.g., Rosenbaum, Chapman, Weigelt, Weiss, \& van der Wel, 2012), inhibition of return (e.g., Tipper, Driver, \& Weaver, 1991), or negative priming (e.g., Tipper, 1985).

Probably the most current, and well-known, example of such effects can be seen in the voluminous work investigating statistical learning (see Saffran \& Kirkham, 2018, for a review), in which observers " ... extract environmental regularities through automatic learning mechanisms operating outside of immediate awareness." (Conway \& Christiansen, 2009 , p. 562). Research on statistical learning has found that this process is relevant to a wide array of domains, including linguistic processing (e.g., Arnon, 2019; Saffran, Aslin, \& Newport, 1996), musical processing (e.g., Jonaitis \& Saffran, 2009; McMullen \& Saffran, 2004; Morgan, Fogel, Nair, \& Patel, 2019), visual processing (e.g., Fiser \& Aslin, 2002; Kirkham, Slemmer, \& Johnson, 2002), tactile processing (e.g., Conway \& Christiansen, 2005), and visuomotor learning (e.g., Cleeremans, 1993; Cleeremans \& McClelland, 1991). These statistical learning processes have been observed to differ across the modalities (Conway \& Christiansen, 2009), and are particularly powerful in the auditory domain, relative to visual and tactile domains (Conway \& Christiansen, 2005). Given all of this evidence, it is clear that the abstraction of global stimulus influences from an experimental context has significant implications for the design and interpretation of experiments cutting across a wide swath of psychological contexts.

\section{Case Study 1: Tonal influences on musical dyad performance}

Schmuckler and Bosman (1997) provide the initial case study in their research on the performance of two-note sequences, or musical dyads. The original goal of this study was to determine if the biomechanical constraints typically observed when typing pairs of letters with two fingers of one hand (2F) versus two hands $(2 \mathrm{H})$ would also be observed when performing pairs of musical notes with one versus two hands. Previous work in typing demonstrated that the time to execute two successive keystrokes was greater for $2 \mathrm{~F}$ relative to $2 \mathrm{H}$ letter pairs (Salthouse, 1986), and that the time to prepare the first keystroke of a pair was greater for $2 \mathrm{H}$ relative to $2 \mathrm{~F}$ keystroke pairs (Sternberg, Monsell, Knoll, \& Wright, 1978). This first result indicates basic biomechanical constraints on overlapping successive finger movements on the same hand, whereas the second result indicates increased planning for $2 \mathrm{H}$ versus 2F letter pairs. Across three experiments, Schmuckler and Bosman (1997) replicated both of these findings within the context of performing $2 \mathrm{~F}$ versus $2 \mathrm{H}$ musical dyads on a piano keyboard. Thus, piano performance induced comparable biomechanical and planning constraints, as did typing.

One aspect of Schmuckler and Bosman's (1997) context was that the stimuli and experimental task did not contain any explicit tonal context. This intention, which was adopted to provide as simple a context as possible, was operationalized by employing sparse musical materials, with no indication of tonality in the presentation of to-be-performed stimuli (a 
musical staff without key signature) and in the actual to-beperformed passages (two musical notes). ${ }^{1}$ Thus, on a trial-bytrial basis, there was little tonal information to be extracted by participants. Because the goal of this work was to compare manual behavior in typing and musical performance, it was important to keep interpretational factors related to musical expression to a minimum, given the work that has demonstrated that inclusion of such factors influences multiple parameters of musical performance, including note duration, note synchrony, and loudness (Gabrielsson, 1999; Palmer, 1989, 1997). Thus, such factors could have undermined the goal of this work, which was to examine preparation and execution times solely on the basis of one- versus two-handed performance.

Despite these precautions, a subsidiary series of analyses provides evidence that participants in these studies abstracted a tonal framework across trials. Data used in these analyses are available via Open Science Framework (https:/osf.io/yf24j/? view_only=be1b3150840e4983a33cd52612565784). In the first two experiments of Schmuckler and Bosman (1997), pianists performed sequential pairs of tones, derived from ten musical notes in all - five each in the left and right hands. The actual notes consisted of the ten contiguous white keys on the piano keyboard, ranging from $\mathrm{E}_{4}$ to $\mathrm{B}_{4}$ in the left hand, and $\mathrm{C}_{5}$ to $\mathrm{G}_{5}$ in the right hand (see Fig. 1). All possible combinations of these ten tones were employed, excluding repetitions of the same note by the same hand (e.g., $\mathrm{E}_{4}$ followed by $\mathrm{E}_{4}$ ); sample stimulus dyads also appear in Fig. 1. Accordingly, a single repetition of all possible combinations (i.e., a block of trials) consisted of 90 trials: 40 stimulus dyads in the $2 \mathrm{~F}$ condition (20 left hand dyads and 20 right hand dyads), and 50 stimulus dyads in the $2 \mathrm{H}$ condition (25 left-right dyads and 25 right-left dyads). Performers completed four blocks of trials, with the first block considered practice.

Experiment 3 extended this paradigm by including all 16 contiguous white and black keys between $\mathrm{E}_{4}$ and $\mathrm{G}_{5}$ (eight keys played by each of the hands). Figure 1 also provides a schematic illustration of this context and sample stimulus dyads. Again, excluding repetitions of the same note by the same hand, this increased number of notes resulted in 240 trials in all: 112 2F stimulus dyads (56 right hand dyads and 56 left hand dyads) and $1282 \mathrm{H}$ stimulus dyads (64 left-right dyads and 64 right-left dyads). Again, performers completed

\footnotetext{
${ }^{1}$ It is important to recognize that presenting a musical staff without any accidentals (e.g., musical sharps [\#] or flats [b]) does suggest two tonalities, C major and A minor, that are indicated by a lack of explicit accidentals. Thus, the goal of not indicating a tonality by not employing an explicit key signature (i.e., a coherent set of accidentals) has the ironic consequence of nevertheless implying two tonalities. In interpreting the impact of this fact, it should be recognized that because all three experiments of Schmuckler and Bosman (1997) presented the same musical staff information, any influences of an implied tonality would be constant across the three experiments. Thus, any differences in findings across these studies suggests that any tonality implied the lack of accidentals had minimal effects on these performances.
}

four blocks of trials, with the first block (shortened to 60 randomly selected trials) considered practice.

How do such stimuli produce a tonal context? Although presented in isolation, any pair of tones can nevertheless imply a larger tonal framework simply by virtue of being sounded. Although such notes are individually tonally ambiguous (e.g., $\mathrm{C}$ and $\mathrm{G}$ are psychologically stable within the tonality of $\mathrm{C}$ major, but are highly unstable within the tonality of F\# major), aggregating across all tones can produce tonal implications for the entire stimulus set. One means of assessing such tonal implications employs the KrumhanslSchmuckler (KS) key-finding algorithm (Krumhansl, 1990; Krumhansl \& Schmuckler, 1986a; Schmuckler \& Tomovski, 2005), which quantifies the fit between the frequency of occurrence or duration of the notes of a stimulus passage and the perceived psychological stability tone ratings for all 24 major and minor tonalities. This algorithm provides a robust assessment of the tonality of a given musical passage in an array of contexts (see Schmuckler, 2016). For these purposes, the KS algorithm was run without any simulation of memory or other temporal contextual influences. Although these constraints are appropriate in the current context (given the nature of the data being analyzed), such factors do offer an interesting opportunity to potentially sharpen the predictions of tonal influences through incorporating memory components.

Table 1 shows the actual frequency of occurrence of the notes within the stimulus sets in Schmuckler and Bosman (1997), as well as the output of the KS algorithm for these stimuli, provided as correlation coefficients. Because Experiments 1 and 2 employed the same stimuli, these studies had identical frequencies of occurrence and KS outputs.

The stimuli of Experiments 1 and 2 had strong tonal implications. Collapsing across octave duplications of notes (e.g., $\mathrm{E}_{4}$ and $\mathrm{E}_{5}$ ), these stimuli consisted of seven distinct notes $-\mathrm{C}$, D, E, F, G, A, and B. Readers with musical training will recognize these notes as the $\mathrm{C}$ major scale, a prototypical representation of a $\mathrm{C}$ major tonality. Not surprisingly, this stimulus set produced the strongest output correlation for a tonality of $\mathrm{C}$ major, $r(10)=.73, p<.001$. Thus, although not intended to instantiate a tonal context, taken as a whole, the trials of this study potentially implied a $\mathrm{C}$ major tonality.

A different picture emerges for Experiment 3. Collapsing across octave duplications of notes reduces these stimuli to 12 distinct tones - C, C\#, D, D\#, E, F, F\#, G, G\#, A, A\#, and B. Again, musically trained readers will recognize this as the chromatic scale, a set that, within a music-theoretic framework, does not imply any specific tonality. Supporting this supposition, the frequency of occurrence values of these stimuli failed to correlate significantly with any major or minor tonality (see Table 1), with a number of keys (E minor, C major, $\mathrm{G}$ major, and $\mathrm{F}$ major) presenting weak, but insignificant correlations. 
Experiments 1 / 2

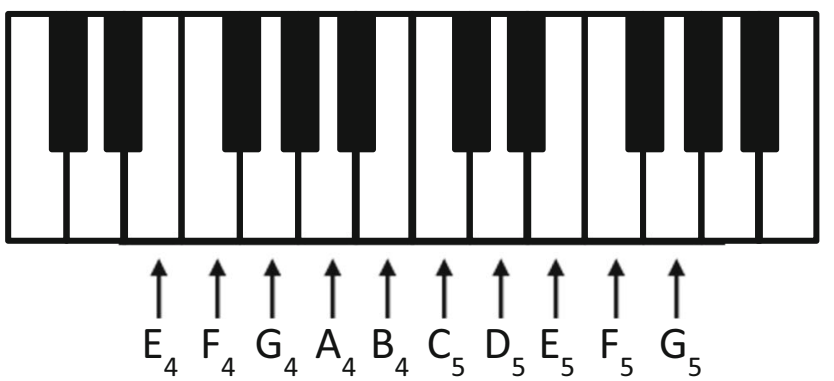

One Hand (2F)

Note 1 Note 2 Left Hand Dyads

$\mathrm{E}_{4}$

$\mathrm{E}_{4}$

$\mathrm{E}_{4}$

$$
\mathrm{F}_{4}
$$

$\mathrm{G}_{4}$

$\mathrm{A}_{4}$

$\mathrm{B}_{4}$

$\mathrm{F}_{4}$

$\mathrm{B}_{4}$

$\mathrm{G}_{4}$

$\mathrm{B}_{4}$

$\mathrm{A}_{4}$

\section{Right Hand Dyads}

$\mathrm{C}_{5}$

$\mathrm{D}_{5}$

$\mathrm{C}_{5}$

$E_{5}$

$\mathrm{C}_{5}$

$\mathrm{F}_{5}$

$E_{5}$

$\mathrm{F}_{5}$

$\mathrm{G}_{5}$
Two Hand (2H)

Note $1 \quad$ Note 2 Left-Right Hand Dyads

$\mathrm{E}_{4}$

$\mathrm{E}_{4}$

$\mathrm{C}_{5}$

$\mathrm{E}_{4}$

$D_{5}$

$E_{5}$

$E_{4}$

$\mathrm{E}_{4}$

$\mathrm{E}_{4}$

\section{Right-Left Hand Dyads}

$C_{5}$

$\mathrm{E}_{4}$

$\mathrm{C}_{5}$

$\mathrm{F}_{4}$

$\mathrm{C}_{5}$

$\mathrm{G}_{4}$

$\mathrm{G}_{5}$

$\mathrm{G}_{5}$

$\mathrm{G}_{5}$
Experiment 3

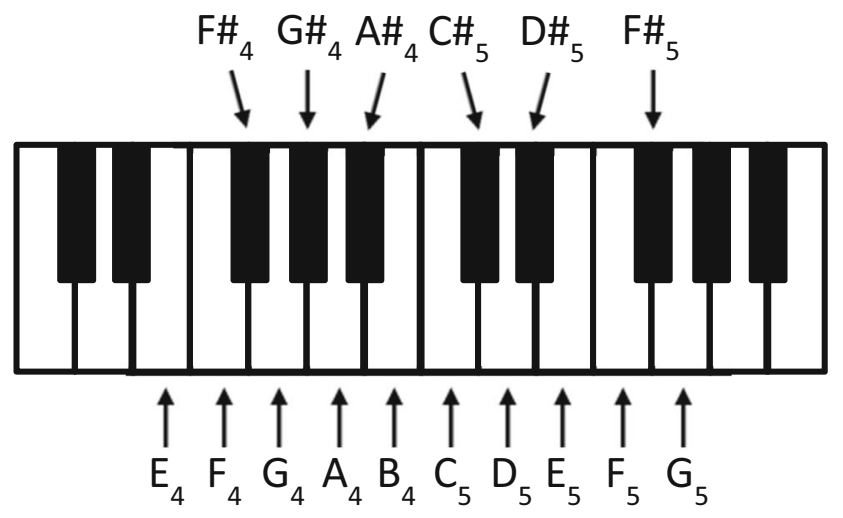

One Hand (2F)

Note 1 Note 2

Two Hand (2H)

Left Hand Dyads

Note 1

Note 2

Left-Right Hand Dyads

$\begin{array}{cc}\mathrm{E}_{4} & \mathrm{~F}_{4} \\ \mathrm{E}_{4} & \mathrm{FH}_{4} \\ \mathrm{E}_{4} & \mathrm{G}_{4}\end{array}$

$\mathrm{E}_{4}$

$\mathrm{C}_{5}$

$E_{4}$

$\mathrm{CH}_{5}$

$\mathrm{E}_{4}$

$D_{5}$
$E_{4}$

$E_{4}$

$\mathrm{E}_{4}$

Right-Left Hand Dyads

\section{Right Hand Dyads}

$\mathrm{C}_{5}$

$\mathrm{CH}_{5}$

$\mathrm{C}_{5}$

$\mathrm{E}_{4}$

$\mathrm{C}_{5}$

$D_{5}$

$\mathrm{C}_{5}$

$\mathrm{F}_{4}$

$\mathrm{C}_{5}$

D\#5

$\mathrm{C}_{5}$

$\mathrm{FH}_{4}$

Fig. 1 Stimulus dyads for the three experiments of Schmuckler and Bosman (1997)

If the implied tonalities of these stimuli were to influence performance, one might predict that the time taken to prepare and/or execute the dyads in Experiments 1 and 2 would be related to $\mathrm{C}$ major in some fashion, whereas the dyads of Experiment 3 would neither show any consistent relation with musical tonality, nor show relations with multiple simultaneous tonalities. The logic here is that if some form of abstract tonal framework, or frameworks (given the possibility of multiple simultaneous tonalities, e.g., Krumhansl \& Schmuckler, 1986b) arose from these dyads, it would structure performances such that 
Table 1. Note counts and KS algorithm outputs for the stimulus dyads of Schmuckler and Bosman (1997)

\begin{tabular}{|c|c|c|c|c|c|}
\hline \multicolumn{3}{|c|}{ Note Counts } & \multicolumn{3}{|c|}{ Output correlations of the KS algorithm } \\
\hline Note & Experiments $1 / 2$ & Experiment 3 & Tonality & Experiments $1 / 2$ & Experiment 3 \\
\hline $\mathrm{C}$ & 18 & 30 & C Major & $.73 * *$ & .32 \\
\hline $\mathrm{C} \#$ & 0 & 30 & C\# Major & -.54 & -.08 \\
\hline $\mathrm{D}$ & 18 & 30 & D Major & .20 & .05 \\
\hline D\# & 0 & 30 & D\# Major & -.26 & -.21 \\
\hline $\mathrm{E}$ & 36 & 60 & E Major & .08 & .06 \\
\hline $\mathrm{F}$ & 36 & 60 & F Major & .52 & .14 \\
\hline F\# & 0 & 60 & F\# Major & -.72 & -.02 \\
\hline G & 36 & 60 & G Major & $.57^{\mathrm{A}}$ & .18 \\
\hline $\mathrm{G \#}$ & 0 & 30 & G\# Major & -.45 & -.38 \\
\hline A & 18 & 30 & A Major & .10 & -.06 \\
\hline A\# & 0 & 30 & A\# Major & .07 & -.02 \\
\hline \multirow[t]{13}{*}{ B } & 18 & 30 & B Major & -.30 & .04 \\
\hline & & & C Minor & .03 & -.22 \\
\hline & & & C\# Minor & -.26 & -.12 \\
\hline & & & D Minor & .44 & .03 \\
\hline & & & D\# Minor & -.64 & -.10 \\
\hline & & & E Minor & $.66^{*}$ & .47 \\
\hline & & & F Minor & .14 & .13 \\
\hline & & & F\# Minor & -.30 & .10 \\
\hline & & & G Minor & .18 & .11 \\
\hline & & & G\# Minor & -.38 & -.25 \\
\hline & & & A Minor & .52 & -.01 \\
\hline & & & A\# Minor & -.47 & -.13 \\
\hline & & & B Minor & .07 & -.01 \\
\hline
\end{tabular}

$\mathrm{A} p=.05 * p<.05 * * p<.01$

preparation and/or execution times would be related to the perceived stability of the dyads within this tonality (or tonalities). The most straightforward relation would be that psychologically stable dyads would be prepared and/or executed more quickly than psychologically unstable dyads.

Accordingly, the preparation and execution times for the stimulus dyads (averaged across participants) were correlated with the perceived stability ratings for these same two tones within all major and minor tonalities. Stability ratings for tone pairs were taken from Table 5.1 in Krumhansl (1990, p. 125), which provides perceived relatedness for all possible ordered pairs of tones (excepting the musical unison, which is a repetition of the same note) with reference to $\mathrm{C}$ major and $\mathrm{C}$ minor tonalities. Krumhansl generated these ratings by having six musically trained listeners hear a key-defining context (e.g., an ascending and descending scale), followed by a sequential pair of tones, and then rating how well the second tone followed the first with respect to the previous key context. Relatedness ratings for the remaining tonalities can be produced by transposing the original ratings to other tonics (Krumhansl \& Kessler, 1982).
Table 2 shows these relatedness ratings for the stimulus dyads of Experiments $1 / 2$ and 3, with reference to the 24 major and minor tonalities. Table 3 shows the correlations between the preparation and execution times for the dyads of Schmuckler and Bosman (1997) with these stability ratings. For the first two experiments, psychologically stable dyads within the key of $\mathrm{C}$ major produced shorter preparation times, resulting in significant or marginally significant negative correlations. Experiment 3 presents an even more subtle and intriguing pattern, with preparation times correlating significantly (negatively) with stability ratings from six different tonalities. Interestingly, if one compares the keys producing significant correlations (C major, D major, G major, A major, E minor, and A minor) for preparation times (Table 3) with the correlations of the tonal implications of the stimulus dyads (Table 1), three of these keys align - those for $\mathrm{E}$ minor, $\mathrm{C}$ major, and $\mathrm{G}$ major. Together, these analyses demonstrate that when individual stimulus dyads collectively and clearly imply a given tonality, performers are faster to produce psychologically stable as opposed to unstable dyads. Interestingly, although Experiment 3 could be interpreted as suggesting either 
Table 2. Relatedness ratings (Table 5.1, Krumhansl, 1990) for the stimulus dyads of Schmuckler and Bosman (1997)

\begin{tabular}{|c|c|c|c|c|c|c|c|c|}
\hline Note 1 & Note 2 & C Major & C\# Major & D Major & $\ldots$ & C Minor & C\# Minor & D Minor \\
\hline $\mathrm{E}$ & $\mathrm{F}$ & 4.50 & 3.58 & 2.83 & & 4.92 & 3.50 & 5.17 \\
\hline$E$ & $F \#$ & 3.67 & 4.50 & 5.25 & & 4.08 & 4.42 & 3.25 \\
\hline $\mathrm{E}$ & G & 4.83 & 2.17 & 2.92 & & 4.75 & 3.50 & 4.67 \\
\hline \multicolumn{9}{|l|}{$\ldots$} \\
\hline $\mathrm{B}$ & $\mathrm{F}$ & 2.00 & 2.33 & 1.83 & & 2.58 & 3.00 & 2.67 \\
\hline$B$ & $F \#$ & 3.50 & 4.83 & 3.92 & & 2.75 & 3.67 & 3.58 \\
\hline B & G & 4.33 & 2.33 & 4.33 & & 3.83 & 3.42 & 3.83 \\
\hline \multicolumn{9}{|l|}{$\ldots$} \\
\hline$C$ & C\# & 2.25 & 6.42 & 3.25 & & 4.08 & 6.42 & 4.50 \\
\hline $\mathrm{C}$ & $\mathrm{D}$ & 5.75 & 2.83 & 4.75 & & 5.00 & 4.00 & 5.33 \\
\hline$C$ & $D \#$ & 2.42 & 3.00 & 2.25 & & 5.25 & 4.58 & 3.42 \\
\hline G & $\mathrm{E}$ & 4.83 & 2.67 & 4.58 & & 2.92 & 3.50 & 4.75 \\
\hline G & $\mathrm{F}$ & 4.58 & 3.58 & 3.75 & & 3.58 & 3.33 & 4.50 \\
\hline$G$ & $F \#$ & 3.00 & 3.58 & 5.25 & & 3.83 & 4.33 & 4.00 \\
\hline
\end{tabular}

Note: Non-italic rows represent stimulus dyads for Experiments 1 and 2; italic rows represent stimulus dyads also included in Experiment 3

Table 3. Correlations between preparation/execution times and dyad relatedness ratings (Krumhansl, 1990)

\begin{tabular}{|c|c|c|c|c|c|c|}
\hline \multirow[b]{2}{*}{ Tonality } & \multicolumn{2}{|c|}{ Experiment 1} & \multicolumn{2}{|l|}{ Experiment 2} & \multicolumn{2}{|l|}{ Experiment 3} \\
\hline & Preparation & Execution & Preparation & Execution & Preparation & Execution \\
\hline C Major & $-.31^{* * * *}$ & -.02 & $-.21^{A}$ & .01 & $-.25^{* * * * * *}$ & -.10 \\
\hline C\# Major & .04 & .19 & .05 & .16 & $.22^{* * * *}$ & .08 \\
\hline D Major & -.06 & .01 & -.04 & .04 & $-.25^{* * * * * *}$ & -.13 \\
\hline D\# Major & .00 & .14 & .00 & .13 & $.28^{* * * * *}$ & $.14^{*}$ \\
\hline E Major & .12 & .12 & .05 & .18 & -.09 & -.01 \\
\hline F Major & -.11 & -.04 & -.12 & -.12 & -.03 & .04 \\
\hline F\# Major & .14 & .13 & .08 & .14 & $.16^{*}$ & .03 \\
\hline G Major & -.10 & .09 & -.09 & -.12 & $-.24^{* * * * * *}$ & -.11 \\
\hline G\# Major & .02 & .02 & .01 & .08 & $.25^{* * * * *}$ & $.16^{*}$ \\
\hline A Major & .02 & .03 & .02 & .14 & $-.16^{*}$ & -.05 \\
\hline A\# Major & -.13 & -.08 & -.18 & -.14 & $.16^{*}$ & .11 \\
\hline B Major & .07 & .21 & .08 & $.21^{\mathrm{A}}$ & -.00 & .01 \\
\hline C Minor & -.18 & .07 & -.04 & -.04 & -.02 & $.14^{*}$ \\
\hline C\# Minor & .06 & -.11 & .07 & .09 & .03 & .01 \\
\hline D Minor & -.18 & -.10 & -.15 & -.08 & -.10 & -.00 \\
\hline D\# Minor & -.04 & -.12 & -.03 & .08 & $.22^{* * * * *}$ & .11 \\
\hline E Minor & -.08 & -.03 & -.10 & .01 & $-.20^{\text {***** }}$ & -.01 \\
\hline F Minor & -.15 & -.06 & -.09 & -.10 & .01 & $.15^{*}$ \\
\hline F\# Minor & -.10 & .01 & -.00 & .05 & -.01 & .02 \\
\hline G Minor & -.13 & .06 & -.09 & -.12 & .01 & .10 \\
\hline G\# Minor & .02 & -.03 & -.01 & .08 & $.14^{*}$ & $.13^{\mathrm{A}}$ \\
\hline A Minor & -.06 & .11 & .00 & .17 & $-.18^{* *}$ & -.02 \\
\hline A\# Minor & -.15 & .01 & -.13 & -.11 & $.22^{* * * *}$ & $.18^{* *}$ \\
\hline B Minor & -.02 & .18 & .09 & .15 & -.09 & .07 \\
\hline
\end{tabular}

$\mathrm{A} p=.05 * p<.05 * * p<.01 * * * p<.005 * * * * p<.001$

Note: Significant negative correlations are in bold and italics 
that these dyads were collectively tonally ambiguous, or that they implied multiple simultaneous tonalities, both interpretations are consistent with the idea that aggregate stimulus properties influenced dyad performances. Finally, the fact that this impact of tonal context was limited to initial preparation of dyad performance implies a form of priming response, a common influence of tonality on musical processing (Bharucha \& Stoeckig, 1986; Bigand \& Poulin-Charronnat, 2016; Bigand, Poulin, Tillmann, Madurell, \& D'Adamo, 2003).

These findings are simultaneously compelling and surprising. The fact that the first two experiments independently replicated a complex pattern for dyad performance is compelling evidence that musical context abstracted out of sparse individual stimulus materials can nevertheless influence performance. Also compelling is that this analysis predicted both the occurrence of single tonal influences (Experiments 1 and 2) and either multiple tonal frameworks or an ambiguous tonal framework (Experiment 3). What is surprising is that this tonal context was induced by aggregating stimulus parameters across successive trials of an experimental procedure that was not intended to produce a tonal framework. Fortunately, this influence was orthogonal to the original goals of this study, and thus did not become a critical confounding variable - stable and unstable intervals are represented in both $2 \mathrm{~F}$ and $2 \mathrm{H}$ stimuli, and thus did not differentially affect preparation or execution times as a function of one versus two-handed performance. ${ }^{2}$ The next case study, however, describes a context in which the unintended tonal influences were not so benign.

One alternative explanation for the observed correlations in these studies is that, rather than the tonal structure arising due to some aggregate structure, it is instead the individual stimulus intervals that have tonal implications, and that these tonal

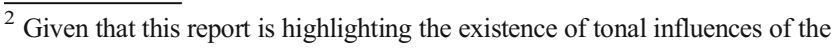
stimulus sets in these experiments, it is important to actually examine whether or not these tonal influences may have (belatedly) played a role in Schmuckler and Bosman's (1997) observed differences between $2 \mathrm{H}$ and $2 \mathrm{~F}$ dyads. In terms of the aggregate intervals, the distributions of frequency of occurrences of notes are, in fact, identical across $2 \mathrm{H}$ and $2 \mathrm{~F}$ conditions, although the exact numbers do vary. However, it is possible that individual intervals evoked tonal implications, with these individual influences varying across $2 \mathrm{~F}$ and $2 \mathrm{H}$ conditions, and thus potentially influencing preparation and execution times. This possibility can be assessed by correlating dummy codes for each stimulus interval with the stability ratings for the ordered pairs of tones from Krumhansl (1990), used in Case Study 1 (see Table 2). These correlations can then be used to assess the tonal influences of the stimulus intervals by identifying the strongest key correlation overall, which indexes the tonal strength of the interval (regardless of specific tonality), and the number of keys with significant correlations, which indexes the specificity of tonal implications (removing intervals that do not imply any key significantly) for the interval. These measures of tonal strength and tonal specificity for the interval stimuli can then be compared as a function of $2 \mathrm{H}$ versus $2 \mathrm{~F}$ stimulus dyads. Reassuringly, an analysis of these measures failed to reveal any differences in either tonal strength or tonal specificity as a function of $2 \mathrm{H}$ versus $2 \mathrm{~F}$ dyads for the stimuli of Experiments 1 and 2, $t(82)=0.29$ (tonal strength) and $t(47)=-$ 0.18 (tonal specificity), n.s., or for Experiment $3, t(230)=0.58$ (tonal strength) and $t(143)=1.28$ (tonal specificity), n.s. Accordingly, the tonal implications of these intervals do not appear to have been a factor in the original findings of Schmuckler and Bosman (1997).
}

implications then influenced performance measures (i.e., preparation and execution times) for that interval. This argument is particularly problematic for Experiment 3 because if different intervals induced different keys, then the multiple key correlations observed for these data might indicate multiple individual tonal influences, as opposed to an aggregate, ambiguous tonal influence.

To examine this possibility we computed the tonal implications of each individual interval in these studies by creating a set of dummy codes for all possible ordered two note intervals (e.g., $\mathrm{C} \rightarrow \mathrm{C \#}, \mathrm{C} \rightarrow \mathrm{D}, \ldots \mathrm{D} \rightarrow \mathrm{C}, \mathrm{C \#} \rightarrow \mathrm{C}, \ldots \mathrm{B} \rightarrow \mathrm{A \#}$, $\mathrm{B} \rightarrow \mathrm{C}$ ), excluding the unison. In this dummy code scheme, each specific interval (e.g., $\mathrm{C} \rightarrow \mathrm{C} \#$ ) received a value of 1 , and all other 131 intervals (all combinations of the 12 chromatic tones, minus the unison) received a value of 0 . These dummy codes were then correlated with the stability values for ordered tone pairs with reference to major and minor tonalities employed in our previous analyses (i.e., Krumhansl, 1990; Table 5.1, p. 125). These analyses thus produced 24 correlations for each two-note interval, with these correlations representing the tonal implications of the interval in isolation.

Three measures of tonal implications were derived from these correlations: (1) whether there was a significant correlation between the interval and any major or minor tonality; (2) the number of tonalities for which there were significant correlations; and (3) the maximum positive correlation, regardless of its statistical significance. In looking at these measures on their own, it is clear that the individual intervals did, in fact, vary in their potential tonal implications. Some intervals, such as $\mathrm{C} \rightarrow \mathrm{A}$, failed to generate a significant correlation with any key, producing a maximum $r(130)=.101, p=.25$. Other intervals, such as $\mathrm{C} \rightarrow \mathrm{G}$, generated a significant correlation with only a single key (C major), resulting in a maximum $r(130)=.155, p<.05$. Finally, other intervals, such as $\mathrm{C} \rightarrow$ $\mathrm{F}$, produced significant correlations with multiple keys (F major, $\mathrm{F}$ minor, and $\mathrm{Bb}$ major; three was the maximum number of significant key correlations for any single interval), generating a maximum $r(130)=.260, p<.003$ (for F major).

The preparation and execution time measures from Schmuckler and Bosman (1997) were then matched and correlated with these tonal implication measures. The logic underlying this analysis is that, if the tonal implications of the individual intervals drove preparation or execution times, then these measures should be correlated with the performance measures. For the number of significant key correlations, this analysis was restricted to only those intervals that produced a significant key correlation, enabling this variable to then represent decreasing tonal specificity (e.g., more key correlations equates to less specific tonal influences); including intervals with no significant key correlations unfortunately reverses this relation. The results of these analyses can be summarized succinctly - none of these measures significantly correlated with either preparation or execution times across any of these 
studies. In other words, individual tonal implications on an interval-by-interval basis were unrelated to the performance measures. Thus, it was only the aggregated tonal implications of the stimuli that influenced behavior.

\section{Case Study 2: Tonal influences on memory for tones in atonal sequences}

The second case study derives from a pilot project related to Vuvan et al. (2014), who investigated the impact of tonalschematic expectancies on musical memory. In this work, listeners heard short (7- to 8-s) melodies that either did or did not contain a specific target tone (the notes D, D\#, or E). These melodies were then followed by a probe note that was one of the three target notes, and listeners were asked whether or not this probe had occurred in the previous melody. Based on the G major tonality of these melodies, listeners should have produced strong expectations for the occurrence of one of the target tones (the note D), moderate expectations for one of the target tones (the note E), and weak expectations for one of the target tones (the note D\#). Interestingly, memory accuracy when the target tone was presented indicated that both highly expected (D) and highly unexpected (D\#) tones were better remembered than moderately expected (E) tones. In addition, listeners showed "false memory" for highly expected tones (i.e., higher false alarm rates) that did not occur in the melody. Across three experiments, this effect was strongest for melodies drawn from a major tonality, weaker for minor tonality melodies (which are psychologically less stable than major tonalities, see Vuvan et al., 2011; Vuvan \& Schmuckler, 2011), and absent for atonal melodies.

In the current context, it is Experiment 3 of Vuvan et al. (2014) that is relevant to this discussion. This experiment employed atonal melodies intended to avoid any sense of tonality (see Fig. 2), with this lack of tonality verified for each melody using the KS algorithm. Table 4 provides the output of the KS algorithm for five representative melodies of this experiment, showing that these melodies were not strongly tonal on an individual basis.

What the published paper does not describe, however, is that a pilot study for this atonal experiment produced findings

\section{Aggregate Tonal Effects}

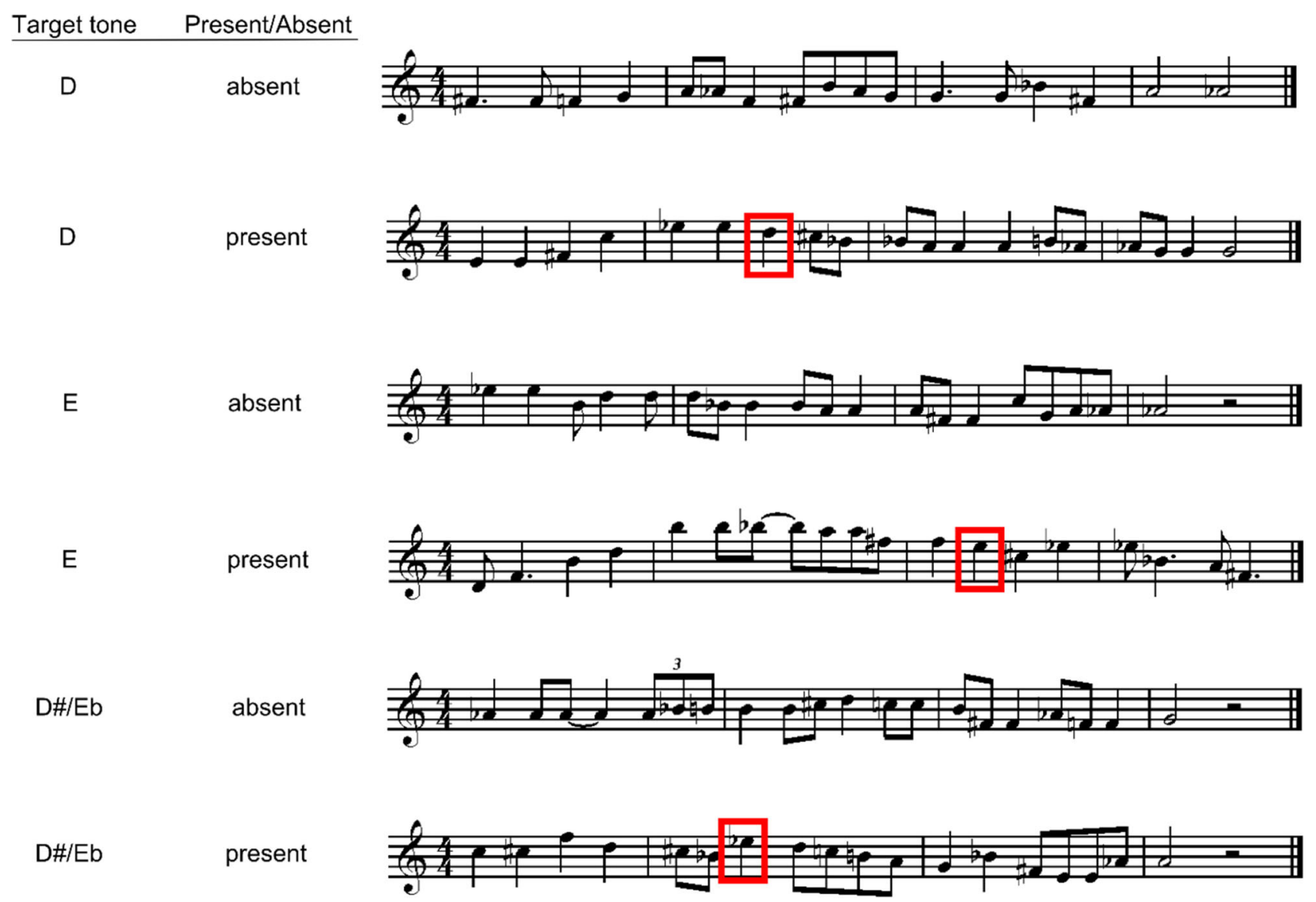

Fig. 2 Examples of melodic stimuli for Experiment 3 of Vuvan, Podolak, and Schmuckler (2014). The boxes indicate present targets 
Table 4. KS correlations between five representative melodies and the 24 key profiles from Experiment 3 (Vuvan et al., 2014)

\begin{tabular}{|c|c|c|c|c|c|}
\hline Key & Melody 1 & Melody 2 & Melody 3 & Melody 4 & Melody 5 \\
\hline $\mathrm{C}$ major & -.03 & .22 & -.44 & -.47 & -.20 \\
\hline $\mathrm{C \#}$ major & .21 & .20 & .54 & .12 & .29 \\
\hline D major & -.06 & .17 & -.02 & .50 & .05 \\
\hline D\# major & -.03 & -.30 & -24 & -.11 & .00 \\
\hline E major & .04 & -.06 & .08 & -.25 & .01 \\
\hline F major & -.19 & -.33 & -.36 & .00 & -.03 \\
\hline F\# major & .06 & .26 & .54 & .25 & -.09 \\
\hline G major & .15 & .43 & .31 & -.17 & -.14 \\
\hline G\# major & .32 & -.10 & .20 & -.20 & .26 \\
\hline A major & -.37 & -.04 & .06 & .30 & -.08 \\
\hline A\# major & -.28 & -.42 & -.15 & .32 & .16 \\
\hline B major & .19 & -.03 & .09 & -.28 & -.24 \\
\hline $\mathrm{C}$ minor & .10 & -.01 & -.29 & -.45 & -.23 \\
\hline $\mathrm{C} \#$ minor & -.14 & .25 & .44 & -.07 & .06 \\
\hline D minor & -.25 & -.30 & .16 & .45 & .30 \\
\hline D\# minor & -.10 & -.32 & .04 & .06 & -.16 \\
\hline E minor & -.09 & .27 & -.40 & -.54 & -.24 \\
\hline F minor & .36 & -.13 & -.01 & -.29 & .39 \\
\hline F\# minor & -.02 & .21 & .26 & .45 & -.07 \\
\hline G minor & -.09 & .17 & -.26 & .21 & .03 \\
\hline G\# minor & .53 & -.11 & .20 & -.26 & .23 \\
\hline A minor & -.24 & -.23 & -.39 & -.09 & -.26 \\
\hline A\# minor & -.26 & -.04 & .43 & .37 & .05 \\
\hline B minor & .20 & .25 & .13 & .15 & -.10 \\
\hline Maximum correlation & .53 & .43 & .54 & .50 & .39 \\
\hline
\end{tabular}

Note. There are no significant correlations, critical $t(10)=.58$. Data for all 72 experimental melodies can be found in the Supplementary Materials

that were curiously anomalous. Data used in these analyses are available via Open Science Framework (https://osf.io/ yf24j/?view_only=be1b3150840e4983a33cd52612565784). Specifically, in this pilot (which employed the exact same methodology as the published paper), participants actually demonstrated better memory performance for the target tone $\mathrm{D}\left(M \mathrm{~d}^{\prime}=0.47, S E=0.08\right)$ than for the target tone D\# $(M=0$. $20, S E=0.15), t(19)=2.02, p=.058$, and the target tone $\mathrm{E}(M$ $=0.18, S E=0.07), t(19)=3.20, p=.005$. Additionally, participants showed higher false-alarm rates to the D\# target tone $(M=0.50, S E=0.03)$ than the D target tone $(M=0.41$, $S E=0.03), t(19)=3.38, p=.003$, and the $\mathrm{E}$ target tone $(M=0$. $40, S E=0.03), t(19)=3.33, p=.004$. These were surprising results given that the atonality of these melodies should have produced equivalent performance across all test tones.

In examining the stimuli for this pilot, one especially enlightening analysis aggregated pitch durations of the melodies across all the stimuli. Although these aggregated durations did not significantly correlate with any given tonality, a close look at the pitch distribution (Table 5) revealed that this distribution was not uniform. This non-uniformity was confirmed with a chi-squared goodness-of-fit analysis, $X^{2}(11)=94.82, p<.001$. Of particular importance was that tones $\mathrm{G}$ and $\mathrm{G} \#$ had the highest durations in this melodic corpus; these notes are highly tonally related (e.g., a perfect fifth relation) to D and D\#, respectively. Previous work has demonstrated that repeated presentation of a single tone is enough to act as a cognitive reference point for inducing a tonal framework (Smith \&
Schmuckler, 2004; Vuvan \& Schmuckler, 2011). Thus, it is very possible that these melodies instantiated representations of $\mathrm{G}$ or $\mathrm{G \#}$ tonalities, leading to responses that then reflected these tonalities. Once the presence of this unintended tonal framework was identified, the stimuli were painstakingly modified to remove this aggregated, potentially emergent tonality, and the experiment was re-run. Using these more globally controlled stimuli, the findings from this study were more interpretable, failing to show differences in recognition as a

Table 5. Distribution of durations of melodies in pilot version of Experiment 3 (Vuvan et al., 2014)

\begin{tabular}{ccl}
\hline Tone & Duration (s) & Notes \\
\hline A & 37.31 & \\
A\# & 52.50 & \\
B & 40.10 & \\
C & 37.09 & \\
C\# & 40.96 & Target tone \\
D & 37.52 & Target tone \\
D\# & 25.05 & Target tone \\
E & 17.90 & \\
F & 22.85 & Duration rank 1 \\
F\# & 70.90 & Duration rank 2 \\
G & 74.02 & \\
G\# & 73.40 & \\
\hline
\end{tabular}


function of the target expectancy, and constitute the data reported in Vuvan et al. (2014).

This second case study is instructive in demonstrating how global stimulus properties can actually work against the explicit intent of an experimental context. Specifically, although the stimuli were tonally ambiguous on an individual, local basis, when taken as an aggregate, they created a tonal framework prevalent enough to produce residual, idiosyncratic findings. Viewed in this fashion, the initially anomalous pilot study results are not only interpretable, they converge with the principal findings of Vuvan et al. (2014). Specifically, the tonal context of the stimuli of the pilot study induced an unintended tonal framework, thereby producing expectations for certain tones, resulting in systematic effects on tone recognition and false-alarm rates. Moreover, and in contrast to the first case study, the unintended influence was particularly detrimental in that it directly undermined a critical manipulation of this study.

\section{Quantifying the growth of aggregate influences}

Given the underlying premise that global influences can develop unbeknownst to an experimenter as a function of aggregate stimulus parameters, it is of interest to attempt to quantify the growth of such influences. Accordingly, for both case studies, a final series of analyses documents how such aggregate effects might occur with stimuli such as these. The general plan of these analyses involved quantifying the tonal implications of the stimuli on a trial-by-trial basis. These tonal implications could then be examined as a function of a cumulative trial model that aggregates such information across increasing trials. Thus, these analyses simulate the growth of tonal implications for participants across sequential trials in these studies, providing a quantification of how such aggregate influences might emerge with exposure.

For Case Study 1, random orders of the experimental trials for Experiments 1 and 2 (number of experimental trials $=90$ )
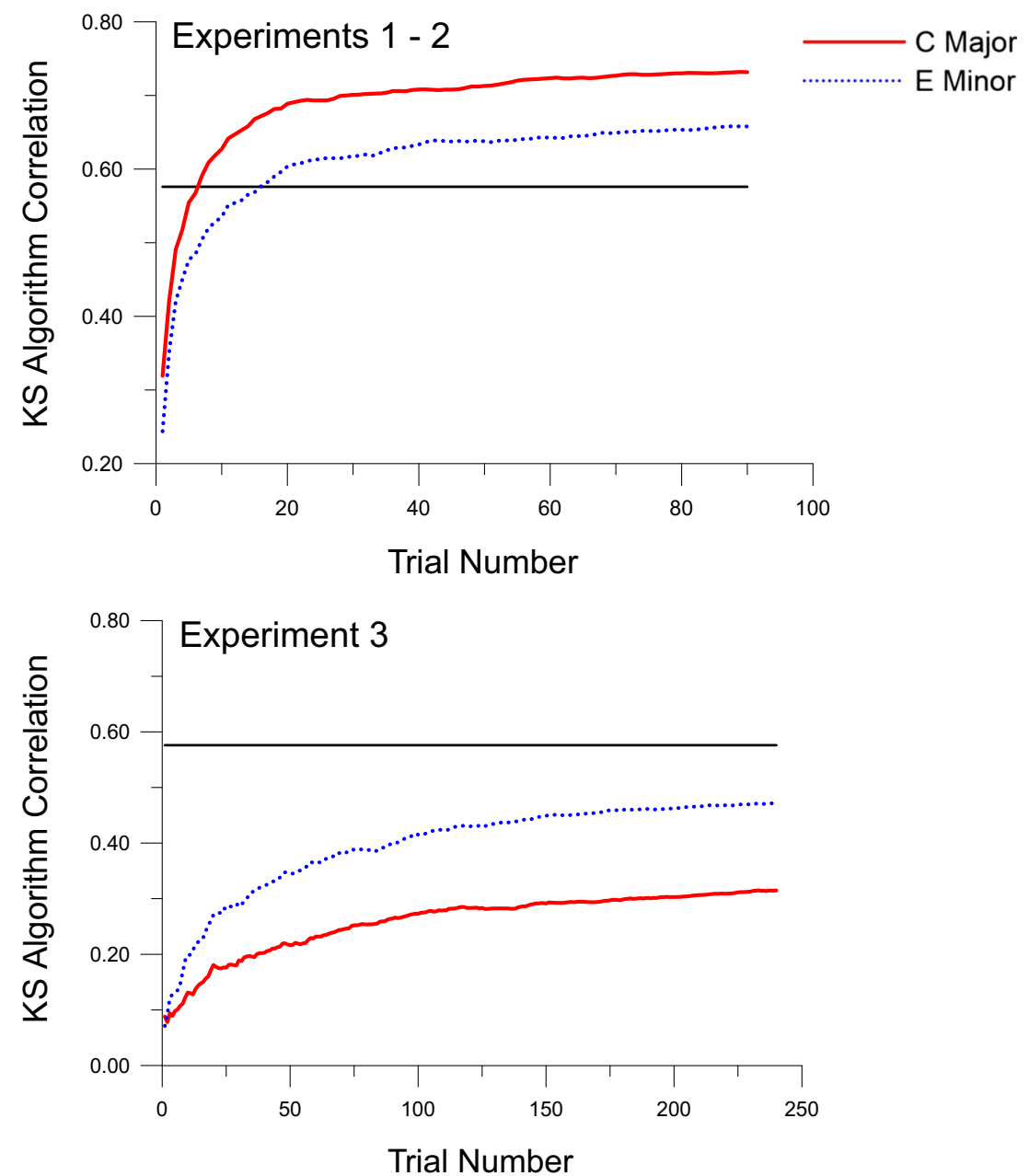

Fig. 3 Cumulative trial simulation $(N=200)$ for the dyad stimuli of Case Study 1 . The .05 significance level is indicated with a horizontal bar 


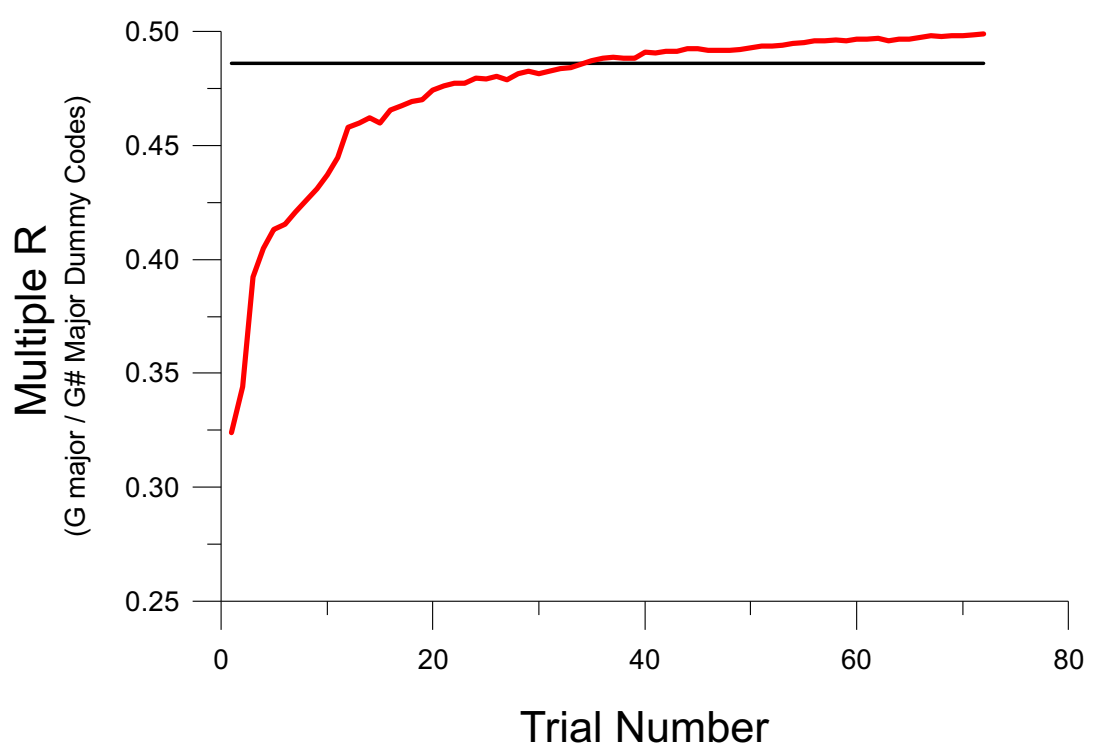

Fig. 4 Cumulative trial simulation $(N=200)$ for the dyad stimuli of Case Study 2. The .05 significance level is indicated with a horizontal bar

and Experiment 3 (number of experimental trials $=240$ ) were created, and frequencies of occurrence note distributions were then constructed based on these random orders. A cumulative trial model of note distributions was created by summing the frequency of occurrence values across increasing trials. For the purposes of this analysis, 200 different random stimulus orderings were constructed, producing 200 cumulative trial models.

For these cumulative trial models, the tonal implications of these frequency of occurrence distributions after each successive trial were analyzed with the KS algorithm. Two candidate keys produced by this algorithm were chosen for examination $-\mathrm{C}$ major and $\mathrm{E}$ minor. These two keys are of interest given that they both showed significant correlations with the aggregated stimuli for Experiments 1 and 2 (see Table 1), and were two of the six significant key correlations for the preparation time data for Experiment 3 (see Table 3). Accordingly, mapping the tonal implications for these stimuli with reference to these tonalities provides insight in the growth of aggregate tonal influences.

Figure 3 shows the averaged (across the 200 different orders) key correlations for $\mathrm{C}$ major and $\mathrm{E}$ minor, as a function of increasing trial. For Experiments 1 and 2, this model exhibits an exponential growth function, inducing $\mathrm{C}$ major quite quickly and becoming statistically significant after seven trials of cumulative information, on average. E minor also demonstrates exponential growth, albeit somewhat slower, requiring 16 trials of cumulative information to produce a significant correlation, on average. The data for Experiment 3 are intriguing, exhibiting both similarities and differences with Experiments 1 and 2 . Comparable to the previous experiments, Experiment 3 also demonstrates exponential growth. ${ }^{3}$ Diverging from these results, however, are that (1) the growth rate for $\mathrm{C}$ major and $\mathrm{E}$ minor are substantially shallower, and (2) this model never produces significant correlations with the candidate keys.

A comparable analysis was conducted for the atonal stimuli described in Case Study 2. For this analysis, the total durations of the notes of the chromatic set (collapsed across octave) were calculated for each melody. These individual melody duration distributions were used to create a series of cumulative distributions as a function of increasing trial (number of experimental trials $=72$ ), employing 200 different random trial orders. These duration distributions were then predicted in a multiple regression, from dummy codes of a $G$ major and G\# major tonality that emphasized the two tonic pitches, $G$ and $\mathrm{G} \#$, by assigning them values of 1 , and the remaining tones with values of 0 . Given the previous explanation of the tonal implications of these melodies underscored this unequal occurrence of these two pitches, this quantification of "tonal strength" was thought to be the most appropriate (see Table 5).

The results of these simulations appear in Fig. 4, which graphs the multiple correlation values across increasing trial. Comparable to the first case study, the cumulative trial model again displays an exponential growth function, although the growth rate of this case study is markedly slower than that of Case Study 1. On average, it requires 35 trials (approximately half a block) to produce a significant multiple correlation.

\footnotetext{
${ }^{3}$ In fact, a subsidiary analysis of all possible major and minor keys for both sets of data revealed that the cumulative model produced either exponential growth or decay functions across trials. Put simply, as more information is aggregated with these stimulus sets, the tonal implications become increasingly differentiated.
} 
Whether this slower aggregation of tonal information is due to the intended (and somewhat unsuccessful) atonal nature of these stimuli, the relative paucity of information in the dummy codes of the two predictor variables, or even arises as a result of fundamental differences in performance versus memory contexts, is unknown. Regardless, it is clear that the aggregate tonal influences in this study were slower to develop, although consistently evident by halfway through a block of experimental trials in this study.

\section{Conclusion}

Together, these case studies underscore the idea that stimulus influences arise not only through one's recognized empirical framework, but also as a result of idiosyncratic, serendipitous, or downright insidious factors. Such influences clearly operate across psychological tasks ranging from basic short-term memory processes to motor planning and production of finger movements, and as such have the potential to be widespread in psychological research. Additionally, the final series of analyses highlights how tonal influences grow over the course of an experimental session. Intriguingly, these case studies demonstrated comparable growth trends, although the time scales of the two sets of data did differ.

As outlined in the Introduction, the impact of global structural components of stimulus sets is not necessarily new, with multiple research domains showing such influences (Lewkowicz et al., 2018; Posner \& Keele, 1968; Rosenbaum et al., 2012; Saffran \& Kirkham, 2018; Tipper et al., 1991). Given the existence of these precedents, what is it about the current formulation that is noteworthy? One aspect that is important in this regard is that in all of these previous examples, investigating participants' abilities to abstract global properties of the presented stimuli was the explicit, intended goal of their respective experimental contexts. In contrast, in the current demonstrations, this abstraction process was an unintended consequence, one that (at best) structured participants' responses in a manner orthogonal to the purposes of the study (Case Study 1), or (at worst) in a manner diametrically opposed to the experimental goals of the experiment (Case Study 2). Thus, these findings are noteworthy in terms of their "accidental" nature within their experimental contexts.

A second aspect that makes these findings important, and one that resonates on a much more general level, is that these findings raise questions about the interpretation of the wellknown "file drawer" problem in research (Franco, Malhotra, \& Simonovits, 2014; Rosenthal, 1979), in which experimental projects "fail" to produce the researchers anticipated or intended results, as well as in the related, and currently much discussed, replication crisis in psychology (Pashler \& Wagenmakers, 2012; Simmons, Nelson, \& Simonsohn, 2011), in which psychological phenomena have been observed to not be replicable; indeed, some authors have raised this issue with specific reference to music psychology research (Frieler, Fischinger, et al., 2013a; Frieler, Müllensiefen, et al., 2013b)

Interestingly, although there has been significant discussion of the replicability issue with respect to questionable research practices (Shrout \& Rodgers, 2018; Simmons et al., 2011; Stroebe \& Strack, 2014) and statistical procedures (Colling \& Szücs, 2018; Szücs \& Ioannidis, 2017; Wagenmakers, Wetzels, Borsboom, \& van der Maas, 2011), much less attention has been paid to the question of what defines a replication in the first place (but see Stroebe \& Strack, 2014, for one such discussion). Simply put, in what manner, on what dimensions, and to what level of detail must an attempted replication mimic the original study to be considered an "acceptable" replication, and thus qualify as providing insight into the vexing issue of whether observed psychological phenomena are indeed replicable? When considering issues of replicability, it is not clear to what extent replication attempts are "required" to employ stimuli that are literally identical to the to-bereplicated study, as opposed to employing conceptually equivalent stimuli intended to embody the same critical information and/or structure, but that are not necessarily identical. One lesson from the current findings is that a potential factor in failures to replicate could lie in unnoticed, unintended abstract properties of stimuli used in such replication attempts employing conceptually (as opposed to identical) stimuli. Accordingly, the process identified in this paper could be playing a role in a perplexing issue that some authors have identified as one of the most important problems currently facing psychology (Bardi \& Zentner, 2017; Trafimow, 2018).

As a final point, one might wonder whether the findings reported here are specific to studies employing musical materials, or are more globally applicable across psychological processing. In addressing such a concern, it is important to note that one of the principle mechanisms underlying the findings in the current analyses involves a tendency to abstract, from a specific set of exemplars, a global framework that is then used in the processing of subsequent information. Framed in this fashion it should be clear that this potential problem will supersede the specific domain of music psychology.

As a specific example of the domain generality of this effect, research on memory for objects has found unintended influences of category structure on spatial memory (Park, Cherry, Smith, \& Lafronza, 1990; Sharps, 1991; Sharps \& Gollin, 1987). Employing comparable paradigms, Sharps and Gollin (1987) and Park et al. (1990) found divergent results in a spatial memory task for a set of household items. Specifically, Sharps and Gollin found an interaction between the age of the participants employed in their study, and the visual detail of the context, such that the recall of elderly individuals was facilitated in environments containing greater 
visual detail, whereas younger adults produced equivalent performance across varying contexts. In contrast, Park et al. failed to observe this same interaction in an analogous study. Subsequent analyses of the actual items used in these two tasks, which were " ... selected as a random assortment of household items" (Sharps, 1991, p. 309), revealed that the sets of items employed by these two research teams actually varied in the degree of their inter-related category structure, with items from Park et al. more highly categorized than the items employed by Sharps and Gollin. Sharps (1991), in a follow-up study, confirmed that this unintended effect of the categorical stimulus structure did indeed underlie the difference in these results. Interestingly, Parks et al. provided the category structure to their stimulus set not as an explicit empirical manipulation, but rather as a means to facilitate counterbalancing of object locations for their spatial memory task. Ultimately, this example thus highlights that unintended abstract stimulus properties can be a concern outside of the narrow domain of music research, significantly influencing one's findings and generating anomalous results in such domains. To our minds, this simple example is symptomatic of the potentially more significant issues underlying such effects.

In conclusion, the current analyses argue that regardless of how carefully one believes they have controlled their stimulus contexts, and have searched for "confounding" factors within their design, there remains the ever-present possibility of emergent stimulus properties arising from the global experimental context. Such properties could have benign effects, or more significantly detrimental implications, for the questions under investigation. As such, researchers must continuously consider multiple levels of structure within their paradigms, including factors that might only emerge in the aggregate.

Author Note This research was supported by a grant awarded to Mark A. Schmuckler from the Natural Sciences and Engineering Research Council of Canada.

Open Practices Statement All data described in this manuscript are available via Open Science Framework (https://osf.io/yf24j/? view_only=be1b3150840e4983a33cd52612565784).

Author Contributions The data for case study 1 were collected, analyzed, and published by M. A. Schmuckler and E. L. Bosman. The data for case study 2 were collected by D. T. Vuvan, O. Podolak, and M. A. Schmuckler, analyzed by D. T. Vuvan and O. Podolak, and published by D. T. Vuvan, O. Podolak, and M. A. Schmuckler. M. A. Schmuckler was responsible for writing the original draft of this manuscript. All authors contributed to subsequent revisions of this work, and all authors approved the final version of the manuscript

\section{References}

Albouy, P., Schulze, K., Caclin, A., \& Tillman, B. (2013). Does tonality boost short-term memory in congenital amusia? Brain Research, 1537, 224-232. https://doi.org/10.1016/j.brainres.2013.09.003
Arnon, I. (2019). Statistical learning, implicit learning, and first language acquisition: A critical evaluation of two developmental predictions. Topics in Cognitive Science, 11, 504-519. https://doi.org/10.1111/ tops. 12428

Bardi, A., \& Zentner, M. (2017). Grand challenges for personality and social psychology: Moving beyond the replication crisis. Frontiers in Psychology, 8. https://doi.org/10.3389/fpsyg.2017.02068

Bharucha, J. J., \& Stoeckig, K. (1986). Reaction time and musical expectancy. Journal of Experimental Psychology: Human Perception and Performance, 12, 403-410.

Bigand, E., \& Pineau, M. (1997). Global context effects on musical expectancy. Perception \& Psychophysics, 59, 1098-1107.

Bigand, E., Poulin, B., Tillmann, B., Madurell, F., \& D'Adamo, D. A. (2003). Sensory versus cognitive components in harmonic priming. Journal of Experimental Psychology: Human Perception and Performance, 29, 159.

Bigand, E., \& Poulin-Charronnat, B. (2016). Tonal cognition. In S. Hallam, I. Cross \& M. Thaut (Eds.), The Oxford handbook of music psychology (2nd ed., pp. 95-111). Oxford, UK: Oxford University Press.

Chew, E. (2014). Mathematical and computational modeling of tonality. New York: Springer.

Cleeremans, A. (1993). Mechanisms of implicit learning: A connectionist model of sequence processing. Cambridge, MA: MIT Press.

Cleeremans, A., \& McClelland, J. L. (1991). Learning the structure of event sequences. Journal of Experimental Psychology: General, 120, 235-253.

Colling, L. J., \& Szücs, D. (2018). Statistical inference and the replication crisis. Review of Philosophy and Psychology, 1-27. https://doi.org/ 10.1007/s13164-018-0421-4

Conway, C. M., \& Christiansen, M. H. (2005). Modality-constrained statistical learning of tactile, visual, and auditory sequences. Journal of Experimental Psychology: Learning, Memory, and Cognition, 31, 24-39. https://doi.org/10.1037/0278-7393.31.1.24

Conway, C. M., \& Christiansen, M. H. (2009). Seeing and hearing in space and time: Effects of modality and presentation rate on statistical learning. European Journal of Cognitive Psychology, 21, 561580. https://doi.org/10.1080/09541440802097951

Fiser, J., \& Aslin, R. N. (2002). Statistical learning of new visual feature combination by infants. Proceedings of the National Academy of Sciences, 99, 15822-15826.

Franco, A., Malhotra, N., \& Simonovits, G. (2014). Publication bias in the social sciences: Unlocking the file drawer. Science, 345, 1502 1505. https://doi.org/10.1126/science.1255484

Frieler, K., Fischinger, T., Schlemmer, K., Lothwesen, K., Jakubowski, K., \& Müllensiefen, D. (2013a). Absolute memory for pitch: A comparative replication of Levitin's 1994 study in six European labs. Musicae Scientiae, 17, 334-349. https://doi.org/10.1177/ 1029864913493802

Frieler, K., Müllensiefen, D., Fischinger, T., Schlemmer, K., Jakubowski, K., \& Lothwesen, K. (2013b). Replication in music psychology. Musicae Scientiae, 17, 265-276. https://doi.org/10.1177/ 1029864913495404

Gabrielsson, A. (1999). The performance of music. In D. Deutsch (Ed.), The psychology of music (2nd ed., pp. 501-602). New York, NY: Academic Press.

Halpern, A. R., Bartlett, J. C., \& Dowling, W. J. (1998). Perception of mode, rhythm and contour in unfamiliar melodies: Effects of age and experience. Music Perception, 15, 335-355.

Jebb, A. T., \& Pfordresher, P. Q. (2016). Exploring perception-action relations in music production: The asymmetric effect of tonal class. Journal of Experimental Psychology: Human Perception and Performance, 42, 658-670. https://doi.org/10.1037/xhp0000172

Jonaitis, E. M., \& Saffran, J. R. (2009). Learning harmony: The role of serial statistics. Cognitive Science, 33, 951-968. https://doi.org/10. 1111/j.1551-6709.2009.01036.x 
Kirkham, N. Z., Slemmer, J. A., \& Johnson, S. P. (2002). Visual statistical learning in infancy: Evidence for a domain general learning mechanism. Cognition, 83, B35-B42.

Krumhansl, C. L. (1990). Cognitive foundations of musical pitch. London: Oxford University Press.

Krumhansl, C. L., \& Kessler, E. J. (1982). Tracing the dynamic changes in perceived tonal organization in a spatial representation of musical keys. Psychological Review, 89, 334-368.

Krumhansl, C. L., \& Schmuckler, M. A. (1986a). Key-finding in music: An algorithm based on pattern matching to tonal hierarchies. Paper presented at the 19th annual Mathematical Psychology Meeting, Cambridge, MA.

Krumhansl, C. L., \& Schmuckler, M. A. (1986b). The Petroushka chord: A perceptual investigation. Music Perception, 4, 153-184.

Lewandowska, O. P., \& Schmuckler, M. A. (2019). Tonal and textural influences on musical sight-reading. Psychological Research. https://doi.org/10.1007/s00426-019-01187-1

Lewkowicz, D. J., Schmuckler, M. A., \& Mangalindan, D. M. J. (2018). Learning of hierarchical serial patterns emerges in infancy. Developmental Psychobiology, 60, 243-255. https://doi.org/10. 1002/dev.21614

McMullen, E., \& Saffran, J. R. (2004). Music and language: A developmental comparison. Music Perception, 21, 289-311. https://doi.org/ 10.1525/mp.2004.21.3.289

Morgan, E., Fogel, A., Nair, A., \& Patel, A. D. (2019). Statistical learning and Gestalt-like principles predict melodic expectations. Cognition, 189, 23-34. https://doi.org/10.1016/j.cognition.2018.12.015

Palmer, C. (1989). Mapping musical thought to musical performance. Journal of Experimental Psychology: Human Perception and Performance, 15, 301-315.

Palmer, C. (1997). Music performance. Annual Review of Psychology, 48, $115-138$

Palmer, C., \& van de Sande, C. (1993). Units of knowledge in musical performance. Journal of Experimental Psychology: Learning, Memory, and Cognition, 19, 457-470.

Park, D. C., Cherry, K. E., Smith, A. D., \& Lafronza, V. N. (1990). Effects of distinctive context on memory for objects and their locations in young and older adults. Psychology and Aging, 5, 250-255.

Pashler, H., \& Wagenmakers, E.-J. (2012). Editors introduction to the special section on replicability in psychological sciences. Perspectives on Psychological Science, 7, 528-530. https://doi. org/10.1177/1745691612465253

Posner, M. I., Goldsmith, R., \& Welton, K. E. Jr. (1967). Perceived distance and the classification of distorted patterns. Journal of Experimental Psychology, 73, 28-38.

Posner, M. I., \& Keele, S. W. (1968). On the genesis of abstract ideas. Journal of Experimental Psychology, 77, 353-363.

Restle, F. (1970). Theory of serial pattern learning: Structural trees. Psychological Review, 77, 481-495.

Rosenbaum, D. A., Chapman, K. M., Weigelt, M., Weiss, D. J., \& van der Wel, R. (2012). Cognition, action, and object manipulation. Psychological Bulletin, 138, 924-946. https://doi.org/10.1037/ a0027839

Rosenthal, R. (1979). The "file drawer problem" and the tolerance for null results. Psychological Bulletin, 86, 638-641.

Saffran, J. R., Aslin, R. N., \& Newport, E. L. (1996). Statistical learning by 8-month-old infants. Science, 274, 1926-1928.

Saffran, J. R., \& Kirkham, N. Z. (2018). Infant statistical learning. Annual Review of Psychology, 69, 181-203. https://doi.org/10.1146/ annurev-psych-122216-011805

Salthouse, T. A. (1986). Perceptual, cognitive, and motoric aspects of transcription typing. Psychological Review, 99, 303-319.
Schmuckler, M. A. (1989). Expectation in music: Investigation of melodic and harmonic processes. Music Perception, 7, 109-150.

Schmuckler, M. A. (1990). The performance of global expectations. Psychomusicology, 9, 122-147.

Schmuckler, M. A. (2004). Pitch and pitch structures. In J. Neuhoff (Ed.), Ecological Psychoacoustics (pp. 271-315). San Diego, CA: Academic Press.

Schmuckler, M. A. (2016). Tonality and contour in melodic processing. In S. Hallam, I. Cross \& M. Thaut (Eds.), The Oxford handbook of music psychology (2nd ed., pp. 143-165). Oxford, UK: Oxford University Press.

Schmuckler, M. A., \& Boltz, M. (1994). Harmonic and rhythmic influences on musical expectancy. Perception \& Psychophysics, 56, 313-325.

Schmuckler, M. A., \& Bosman, E. L. (1997). Interkey timing in piano performance and typing. Canadian Journal of Experimental Psychology, 51, 99-111.

Schmuckler, M. A., \& Tomovski, R. (2005). Perceptual tests of an algorithm for musical key-finding. Journal of Experimental Psychology: Human Perception and Performance, 31, 1124-1149.

Sharps, M. J. (1991). Spatial memory in young and elderly adults: Category structure of stimulus sets. Psychology and Aging, 6, 309-312.

Sharps, M. J., \& Gollin, E. S. (1987). Memory for object locations in young and elderly adults. Journal of Gerontology 42, 336-341.

Shrout, P. E., \& Rodgers, J. L. (2018). Psychology, science, and knowledge construction: Broadening perspectives from the replication crisis. Annual Review of Psychology, 69, 487-510. https://doi.org/10. 1146/annurev-psych-122216-011845

Simmons, J. P., Nelson, L. D., \& Simonsohn, U. (2011). False-positive psychology: Undisclosed flexibility in data collection and analysis allows presenting anything as significant. Psychological Science, 22, 1359-1366. https://doi.org/10.1177/0956797611417632

Smith, N. A., \& Schmuckler, M. A. (2004). The perception of tonal structure through the differentiation and organization of pitches. Journal of Experimental Psychology: Human Perception and Performance, 30, 268-286.

Sternberg, S., Monsell, S., Knoll, R. L., \& Wright, C. E. (1978). The latency and duration of rapid movement sequences: Comparisons of speech and typewriting. In G. E. Stelmach (Ed.), Information processing in motor control and learning (pp. 117-152). New York: Academic Press.

Stroebe, W., \& Strack, F. (2014). The alleged crisis and the illusion of exact replication. Perspectives on Psychological Science, 9, 59-71. https://doi.org/10.1177/1745691613514450

Szücs, D., \& Ioannidis, J. P. A. (2017). When null hypothesis significance testing is unsuitable for research: A reassessment. Frontiers in Human Neuroscience, 11. https://doi.org/10.3389/fnhum.2017. 00390

Thompson, W. F., \& Mor, S. (1992). A perceptual investigation of polytonality. Psychological Research, 54, 60-71.

Tillman, B., Bharucha, J. J., \& Bigand, E. (2000). Implicit learning of tonality: A self-organizing approach. Psychological Review, 107, 885-913.

Tipper, S. P. (1985). The negative priming effect: Inhibitory priming by ignored objects. The Quarterly Journal of Experimental Psychology, $37 A, 571-590$

Tipper, S. P., Driver, J., \& Weaver, B. (1991). Object-centred inhibition of return of visual attention. The Quarterly Journal of Experimental Psychology, 43A, 289-298.

Trafimow, D. (2018). An a priori solution to the replication crisis. Philosophical Psychology, 31, 1188-1214. https://doi.org/10.1080/ 09515089.2018.1490707 
Vuvan, D. T., Podolak, O. M., \& Schmuckler, M. A. (2014). Memory for musical tones: The impact of tonality and the creation of false memories. Frontiers in Psychology: Auditory Cognitive Neuroscience, 5, 1-18. https://doi.org/10.3389/fpsyg.2014.00582

Vuvan, D. T., Prince, J. B., \& Schmuckler, M. A. (2011). Probing the minor tonal hierarchy. Music Perception, 28, 461-472.

Vuvan, D. T., \& Schmuckler, M. A. (2011). Tonal hierarchy representations in auditory imagery. Memory \& Cognition, 39, 477-490.
Wagenmakers, E.-J., Wetzels, R., Borsboom, D., \& van der Maas, H. L. J. (2011). Why psychologists must change the way they analyze their data: The case of Psi: Comment on Bem (2011). Journal of Personality and Social Psychology, 2011, 426-432. https://doi.org/ 10.1037/a0022790

Publisher's note Springer Nature remains neutral with regard to jurisdictional claims in published maps and institutional affiliations. 